\title{
A Direct Torque Controlled Anchor System Design
}

\author{
Huilue Jiang1,2, Fei Luo1,2 \\ ${ }^{1}$ Shandong Academy of Sciences Institute of Oceanographic Instrumentation, Qingdao, 266001, \\ China \\ ${ }^{2}$ Shandong Provincial Key Laboratory of Ocean Environment Monitoring Technology, Qingdao, \\ 266001, China
}

Keywords: Anchor, direct torque, fuzzy; PID.

\begin{abstract}
The anchor is the core component of vessel mooring system, of which an accurate and efficient control mechanism plays important roles in ensuring the optimal operation conditions. For the limit in reliability and automation, traditional relay logic control is gradually unable to meet the requirement of modern anchor control system. While using transducer as the drive mechanism of anchor with direct torque control would achieve a wide range of speed modulation, and greatly improve the performance of auxiliary engines. In the model design, PLC is taken as the controller. Fuzzy and PID control is introduced to realize anchor speed control according to the variation of loading torque in different working stages. The simulation results show that the revolving speed can be increased in under loading conditions, thereby the efficiency of weigh anchor can be improved.
\end{abstract}

\section{Introduction}

As the core component of mooring system, anchor is mainly used to deploy and retract the anchor chain, which plays an important role in the normal operation and emergency situations[1]. In normal circumstances, vessels can be fixed by anchor system in safety area for a relatively long time operating or berthing; for the emergency situation such as stranded or maneuver capacity losing, anchor system can be used to prevent the unexpected shift in waves flow impact. In addition, anchor system can also be used to assist the forward, backward and turning around actions in exceptional circumstances. By the direction of the chain axle centerline, anchor system can be divided into two different types, namely horizontal and vertical anchor system, where the vertical anchor system with power section fixed below the deck is more used for saving the deck space.

Actually the anchor systems require a speed control range for operation, which are high speed and low load for the mooring state and low speed and high load for anchor breaking state[2]. For the traditional electric anchor system, power frequency voltage is directly applied to the wound multi-speed motor, as a result the speed control range is limited and motor structure is complex and bulky. The control loop of the anchor system mainly includes contactors, thermal relays, cam switches, time relays and other auxiliary devices, in which the cam switches, achieve the motor positive-negative rotating and low, medium, high operating speed[3,4]. This traditional relay logic control could achieve the requirements of the basic control function with a low price, but disadvantaged in reliability and automation. As the developing of automation technology and equipments, the drive mechanism turns to inverter which controls the speed by direct torque control system, and controller adopts PLC for increased automation.

\section{System hardware design}

The hardware part of anchor system consists of the operating mechanism, control PLC, marine inverter, inverter motor, speed encoder, anchor mechanical devices, brake contactor, brake motors and torque sensors, in which operation mechanism and PLC is direct connected by I/O hard wired, PLC and marine inverter is direct connected by I/O hard wired and Profibus-DP, marine inverter and inverter motor is connected by wire, marine inverter and brake motors is connected by brake contactor, inverter motor is mechanically connected to the anchor mechanical devices and also connected to the marine inverter by speed encoder which feedback the speed signal to the marine 
inverter. The force moment of the anchor is detected by moment sensor and output to the PLC as $4 \sim 20 \mathrm{~mA}$ analog signal. A typical mooring system is shown in Fig.1.

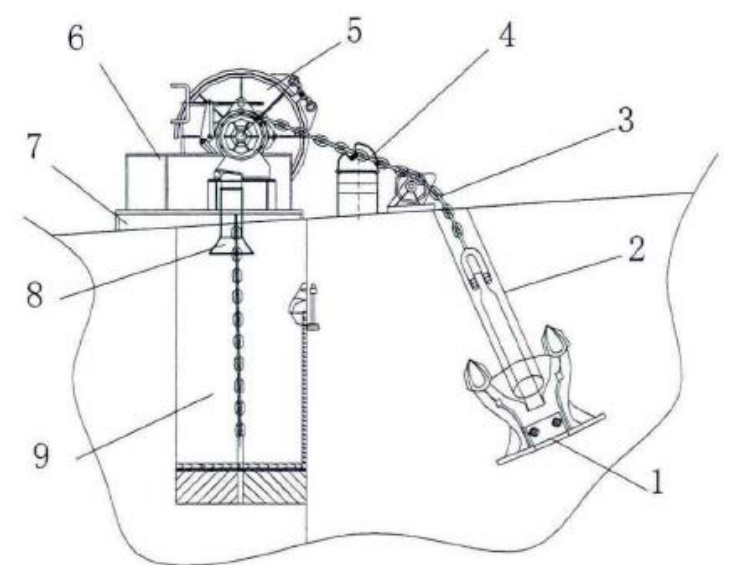

Fig.1 A typical mooring system configuration

1, anchors; 2, anchor chains cylinder; 3, chain; 4, the chain engine; 5, windlass; 6 , windlass base; 7 , the support structure; 8, anchor tube; 9, chain lockers

The inverter used here is ACS 800 series drives of ABB. As an AC inverter based direct torque control, this inverter could precisely control the speed and torque of the inverter motor, which could be applied to the lifting equipment as anchor system. When using direct torque control, each individual inverter could be taken to obtain the value of stator flux and torque, and then realize the switching control. The control method of direct torque control is different from PWM vector control which obtains the value of magnetic flux by pre-estimate the flux state. So the control speed of direct torque control is faster.

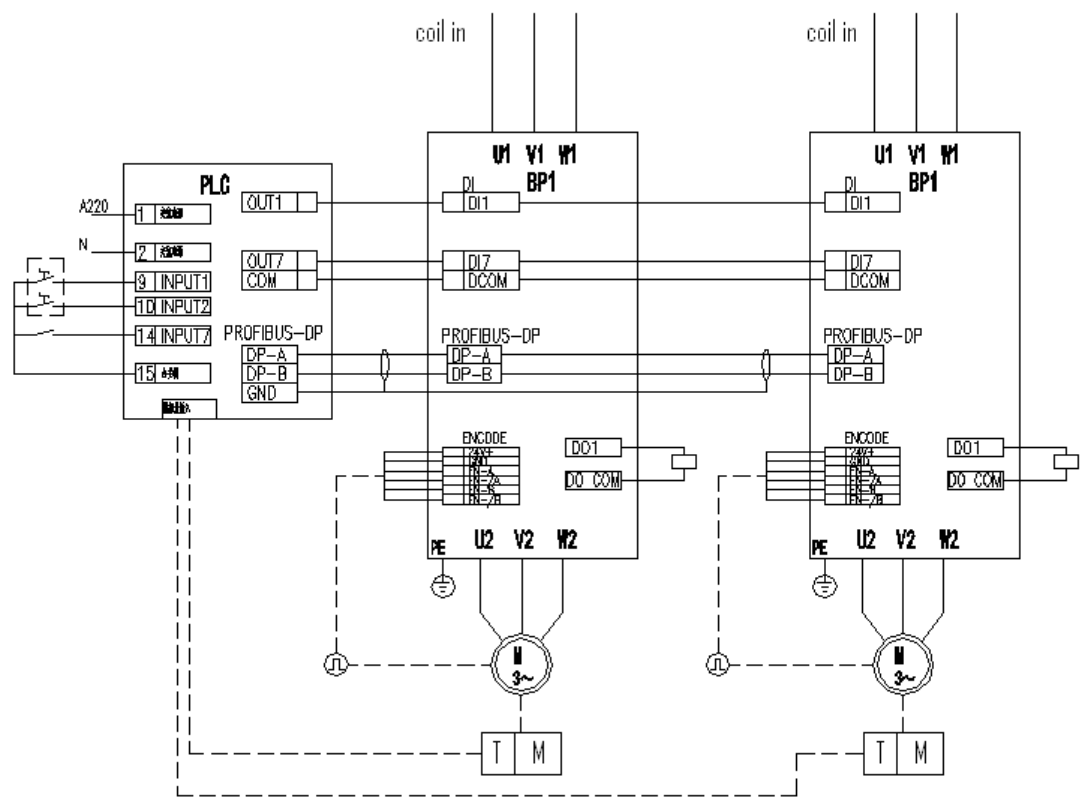

Fig.2 Anchor hardware system diagram

ACS 800 series inverter can select multiple given parameters[5]. In order to improve the performance of the system, here we chose the hybrid control mode that includes direct I/O mode and communication mode. In the hybrid control mode, we can control the starting, ending and direction of DI inverter and torque of $\mathrm{AI}$ inverter by direct $\mathrm{I} / \mathrm{O}$, and setting the value of inverter speed real time by Profibus-DP to realize the torque and speed controlling. Profibus-DP used here could achieve accessibility of the field data in the process of production, which covers the requirement of communication in the field of sensor/actuator and capable of network communication in the field of unit that has become the de facto internationally standards.

The controller used here is Siemens CPU 224XP CN which has been widely used in the automation field of detecting, monitoring and controlling. The advantages of this controller mainly 
include good reliability, extensive instruction set and built-in integration capability, real time characteristics, communication capabilities and extensions. It has 24 digital I/O data channels that 14 for input and 10 for output, 3 analog I/O data channels that 2 for input and 1 for output. Both the digital and analog channels can be expanded, where the maximum channel is 168 for digital and 38 for analog. Meanwhile, the controller has $22 \mathrm{~K}$ storage space, 6 independent $100 \mathrm{KHz}$ high speed counter input, $2100 \mathrm{KHz}$ high speed impulse output, 2 RS485 interfaces, and supporting PPI, MPI communication protocols. This controller also has some additional features such as built-in analog I/O, position control features, self-tuning PID function, linear ramp pulse command, diagnostic LED, data logging and recipe functions. The expansion module used here is EM277 PROFIBUSDP which is taken to connect the bus interface of inverter.

\section{System software solutions}

The control of vessel anchor mainly refers to anchor weighing and dropping. Anchor dropping is a relatively simple operation that can be realized by motor reversely rotating and speed adjustment. Compared with anchor dropping, anchor weighing is a relatively complex process. In the process of anchor weighing, the force of anchor varies constantly[6]. A typical process of anchor weighing can be divided into 4 stages, and the torque curve is shown in Fig.3.

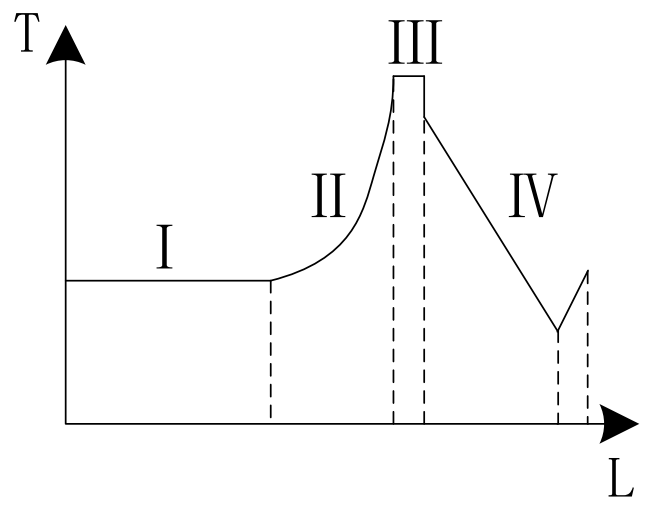

Fig.3 The torque curve in four different working stages

In the first stage, the hanging anchor chain would be collected. The anchor system mainly prevents the shift of vessel induced by wind, waves and water flow. In this stage, the torque of anchor could stay in a certain value and can be described as follow:

$$
\mathrm{F} 1=\mathrm{F}+\rho(0.87 \mathrm{H}+\mathrm{h})
$$

where $\mathrm{F}$ is the sum of resistance suffered from water flow and wind, $\rho$ is the weight of anchor chain per meter, $\mathrm{H}$ is the normal anchor depth, $\mathrm{h}$ is the height of anchor chain centre from sea surface.

In the second stage, anchor chain would remain a straightened state until the anchor breaking through the seabed. In this stage, vessel would right above the anchor, and the resistant torque would gradually increase. The decreasing value of anchor chain is:

$$
12=\mathrm{l}-(\mathrm{H}+\mathrm{h})=[2.3 \mathrm{H} \times \mathrm{F} / \mathrm{\rho}+(\mathrm{H}+\mathrm{h}) 2] 1 / 2-(\mathrm{H}+\mathrm{h})
$$

In this process, it can be approximately considered as a linear relationship between the anchor tension and tension decreasing, so the anchor tension can be described as:

$$
\mathrm{F} 2=\mathrm{k} \times \mathrm{l} 2
$$

In the third stage the anchor would break the seabed. In this stage the resistant torque comes to the maximum and keeps stable. The maximum value of the torque equals to the inertial force of vessel shifting. There are 2 working states of the vessel motor in this stage, one is anchor breaking seabed by the resistant torque, and the other is unable to break the seabed and then turn to the inertia force. According to the first working state of the vessel motor, the tension of the anchor chain can be computed by:

$$
F_{3}=2 G+0.87(G+\rho \times h)+\rho \times h
$$


where $\mathrm{G}$ is the weight of the anchor.

In the fourth stage, the anchor chain would be collected into the anchor position. Owing to the anchor chain shorting, resistant torque would gradually decrease until the chain totally been collected. In the process of anchor collecting, the tension of the anchor chain at beginning and end can be obtained by Eq. (5) and (6).

$$
\begin{gathered}
F_{4}=F_{3}-2 G=0.87(G+\rho \times h)+\rho \times h \\
F_{4}=G
\end{gathered}
$$

By Eq. (1) (6), we can obtain the resistant force of the anchor in different working stages. Considering the environmental factors such as water domain, wind, water flow and water plants, the load of the anchor would be different, so it would be difficult to exactly describe the anchor force by mathematics. On the contrary, fuzzy control and PID control would be a better choice in this situation, by which we can adjust anchor speed in different torque states and finally approve the anchor weighing efficiency.

At the points of stage transferring, the given torque value is set to be the larger, and the anchor speed could be set by the former stage. Then the speed variation would be gently and mechanical shock would be prevented.

\section{Numerical simulation and results analysis}

Here we take a marine research vessel as the prototype to set the relative parameters. The anchor system of this vessel has been rebuilt by the method mentioned above, namely taking direct torque control instead of the original multi-speed wound motor driving, and realizing speed control by cam gear. To test the variation of torque in the process of anchor weighing, a numerical simulation model is established in Matlab. In the simulation model, the given torque in different working stage is obtained by fixed algorithm, and the maximum speed is alternated to direct the best performance of the inverter. At the points of each stage, the given torque is set as the larger value of adjacent stages. The value of the limited speed would change to enhance the ability of anti load mutation. The speed variation curve of simulation is shown in Fig.4.

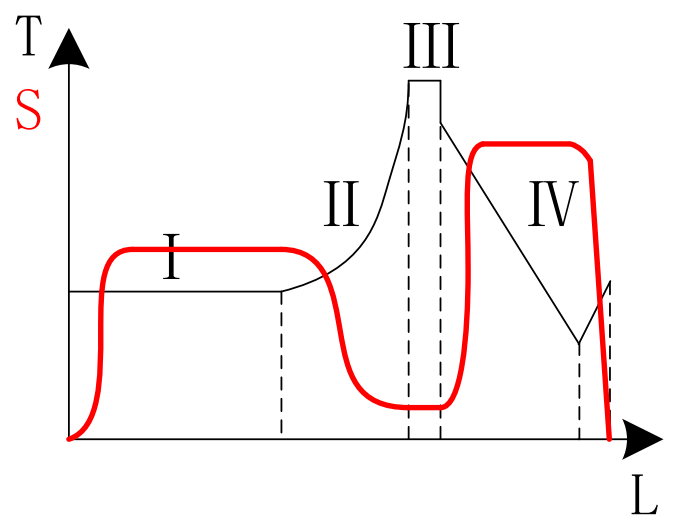

Fig.4 The speed variation curve of simulation

In Fig.4, the red line is the curve of speed, and the black line represents the given resistant force of the anchor. The speed characteristic curve of the rebuilt vessel is shown in Fig.5. 


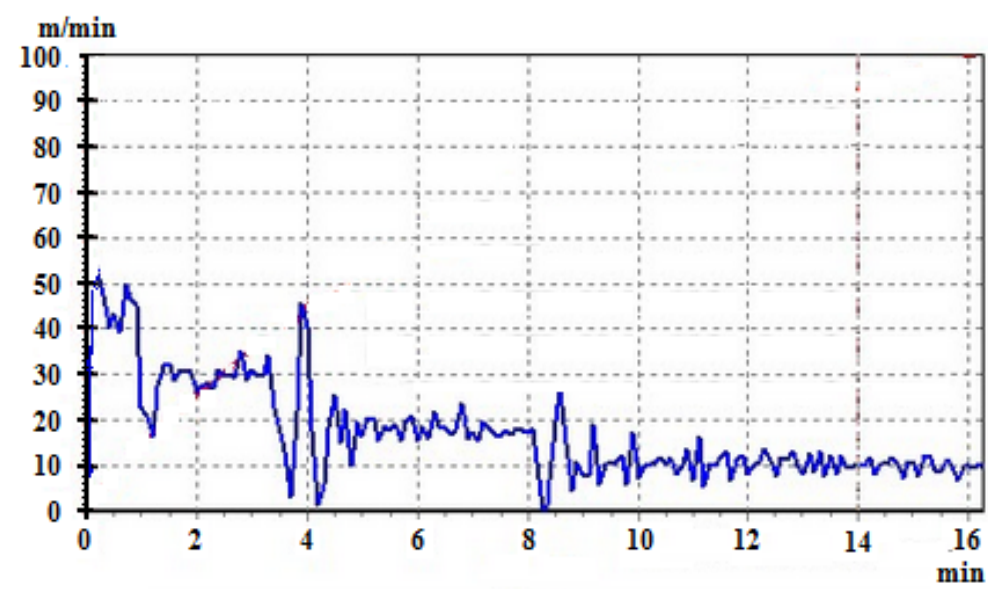

Fig.5 The speed characteristic curve of the rebuilt vessel

From the comparison of Fig.4 and Fig. 5 we cans see, the speed of the anchor can be alternate infinitely variable, and the variation of anchor speed is gently that decrease the mechanical shock. When anchor under loading, the speed of anchor would be improved. And when heavy loading, the speed would be reasonably decreased, which realize a overall efficiency enhancement.

\section{Conclusions}

The hardware part of the anchor system is taken as PLC controlling and inverter driving, where the inverter is controlled by direct torque to realize the infinitely speed adjustment. The software part of the system is realized by fuzzy PID control, which makes the speed variation of anchor system gently and decrease the mechanical shock. By the modification mentioned above, the speed of anchor at under loading situation could be improved and direct the performance enhancement of the anchor system.

\section{Acknowledgements}

The research work was supported by International Science \& Technology Cooperation Program of China under Grant No. 2014DFR60490.

\section{References}

[1] Editorial committee. Handbook of vessel design. Beijing: National defense industry press, 2013.

[2] Zhang Shuzhong, Development of automation control technology for anchor winch. Marine Electric\& Electronic Engineering, 32(4), pp. 46 48, 2012.

[3] Shang Deyong, Pan Yue \& Liu Zhimin, Application of direct torque control system in controlling hoist, Coal Mine Machinery, 30(10), pp.184 186, 2009.

[4] Hu Dong, Liu Yongdong, Direct torque control strategies of induction motor-status and trend. Electric drive, 3, pp.3 8, 2004.

[5] Yang Lei, Liu Xiaofei, \& Gao Min, Multi-motor driven technology based on ABB ACS800. Electrician Technology, 2(11), pp.15 17, 2007.

[6] Ma Nanqi, Research on automatic controlling of marine anchor base on fuzzy algorithm. Journal of Wuhan University of Technology(Transportation Science \& Engineering), 26(3), pp. 362 363, 2002. 